\title{
Characterization and Nanoscale Strain Analysis of Surface Modified Layer in $\mathrm{Y}_{2} \mathrm{O}_{3}$
}

\author{
J.F. Al-Sharab, B.H. Kear, S. Deutsch and S.D. Tse \\ Center for Nanomaterials Research, 607 Taylor Road, Piscataway, NJ 08854-8065
}

Yttrium oxide $\left(\mathrm{Y}_{2} \mathrm{O}_{3}\right)$ is a major component in optical windows and rare-earth doped lasers [1-2]. Recently, nanocrystalline $\mathrm{Y}_{2} \mathrm{O}_{3}$, with grain size $<100 \mathrm{~nm}$, has become the focus of attention because it offers improved mechanical performance while maintaining high optical transmittance in the mid-IR range (2-8 $\mu \mathrm{m})$. Conventional powder consolidation methods require high sintering temperatures, which makes the fabrication of nano-grained structures challenging. To circumvent this problem, we have been investigating a reversible phase transformation (RPT) process, under high pressure, to transform bulk polycrystalline $\mathrm{Y}_{2} \mathrm{O}_{3}$ directly into the nanocrystalline state. So far, starting with coarse-grained $(300 \mu \mathrm{m})$ polycrystalline $\mathrm{Y}_{2} \mathrm{O}_{3}$, a single RPT operation conducted at $1000^{\circ} \mathrm{C}$, has reduced the grain size to $\sim 100 \mathrm{~nm}$ [3]. An unexpected finding was the appearance of a surface modification effect, which increased with holding time at $1000^{\circ} \mathrm{C}$. As will be shown, this effect can be understood in terms of a reductive decomposition mechanism, due to reaction of the $\mathrm{Y}_{2} \mathrm{O}_{3}$ sample with the graphite heater. In what follows, we report on observations made by analytical electron microscopy (AEM) methods, including nano-scale strain analysis.

Disc-shaped samples ( 4 x $4 \mathrm{~mm}$ ) of polycrystalline $\mathrm{Y}_{2} \mathrm{O}_{3}$, prepared by hot isostatic pressing (HIP) of powder compacts, were obtained from Raytheon IDS. The samples were subjected to high pressure-high temperature (HPHT) treatments, using a high pressure unit of novel design. Samples for SEM observation were fractured just before insertion into the microscope to minimize surface reaction with the environment. Thin foils for TEM examination were prepared by the wedge technique. EDS spectral analysis was used to determine the relative intensities of elements present. HRTEM imaging was utilized to analyze phases, dislocation density and strain mapping. Strain analysis was carried out using the Hÿtch method [4].

SEM examination of a fractured sample showed a faceted columnar-grained structure, with cubic symmetry, covering the entire sample surface to a depth of $\sim 20 \mu \mathrm{m}$, Fig. 1. EDS analysis showed that $\mathrm{Y} / \mathrm{O}$ ratio for a smooth intergranular fracture of coarse-grained $\mathrm{Y}_{2} \mathrm{O}_{3}$ is depleted in oxygen relative to a rough transgranular fracture surface. Similarly, the columnar-grained structure is depleted in oxygen relative to the transgranular fracture surface. Hence, grain boundaries in the original cubic- $\mathrm{Y}_{2} \mathrm{O}_{3}$, as well as the surface columnar-grained structure, must have experienced oxygen depletion by reaction with the graphite heater, i.e. by a reductive decomposition mechanism. Progressive reduction of the surface layers, facilitated by relatively fast oxygen diffusion along the directionally-aligned grain boundaries, could explain this behavior. The appearance of a carbon-enriched layer, Fig. 1, in front of the columnar-grained structure indicates rejection of impurity carbon during the directional growth process.

Figs. 2(a)-(c) show filtered maps of HRTEM images showing the presence of many isolated dislocations, as well as dislocation dipoles in the columnar-grained structure. A rough estimate of dislocation density within the columnar grains is $\sim 10^{12} / \mathrm{cm}^{2}$. This high degree of plastic deformation is attributed to thermal expansion mismatch stresses, which affect the softer surface layer more than the harder interior. Based on nanoindentation results, the columnar grains are an order of magnitude softer than sample interior ( 0.6 GPa vs $10 \mathrm{GPa})$. In strain mapping analysis, a strain-free simulated image is used as reference. A typical example of the strain field around an edge dislocation is shown in Fig. 3. As expected, strain across the edge dislocation changes sign from tension to compression. The lack of good HRTEM images from bulk material did not allow a similar estimation of dislocation density.

Further tests are underway to increase the thickness of the columnar-grained surface layer by enhancing grain-boundary diffusion at higher temperatures. A thicker layer should enable a more detailed analysis to correlate structure with optical and thermal properties. 
References:

1. Yong-Nian $\mathrm{Xu}$ et al., Electronic, structural, and optical properties of crystalline yttria, physical review B.,56, No. 23, (1997-I)

2. J.F. Al-Sharab et al., Synthesis and Characterization of Plasma Synthesized Nanostructured Magnesia-Yttria Based Nanocomposites, Mater. Res. Soc. Symp. Proc. Vol. 1056, Materials Research Society 1056-HH08-44, (2008)

3. B.H. Kear et al., On the Conversion of Bulk Polycrystalline $\mathrm{Y}_{2} \mathrm{O}_{3}$ into the Nanocrystalline State, , Journal of the American Ceramic Society (submitted)

4. J. Hÿtch et al., Measurement of the displacement field around dislocations to $0.03 \AA$ by electron microscopy, Nature 423, 270-273 (2003)

5. This work is supported by a grant from the Office of Naval Research
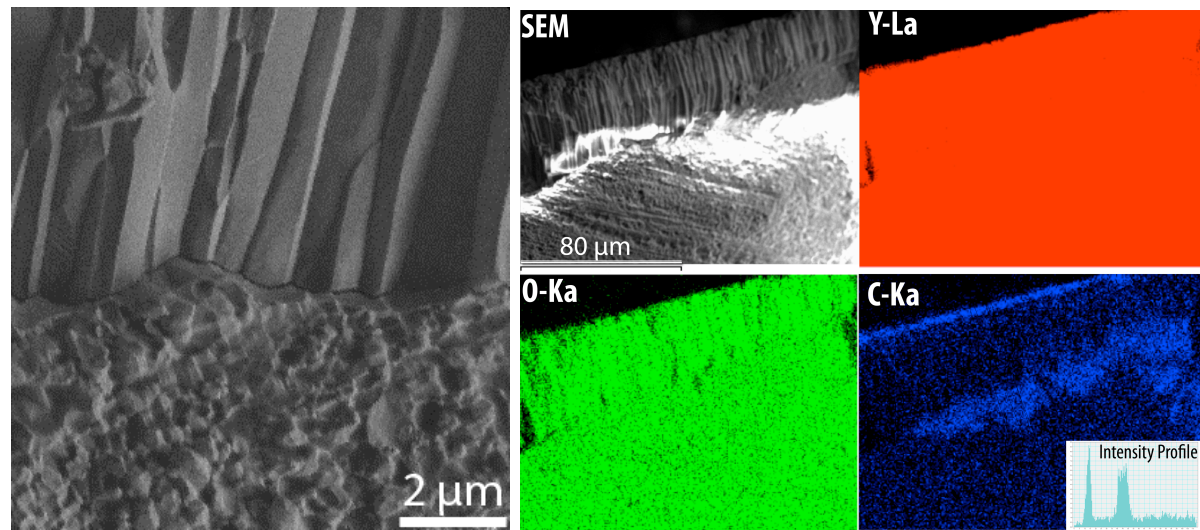

Fig. 1 - SEM examination of a typical fractured sample of $\mathrm{Y}_{2} \mathrm{O}_{3}$ after hot pressing, showing surface columnar grains and EDS chemical mapping.
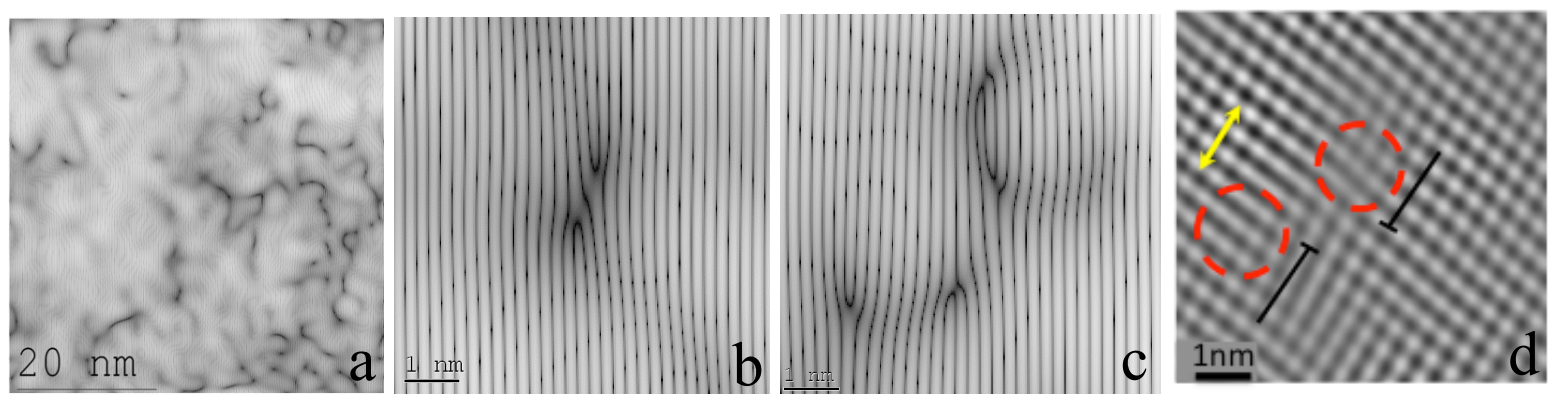

Fig. 2 - Low (a) and high (b-d) magnification filtered HRTEM images showing single and dipole dislocations in columnar grains. Note that each dislocation is dissociated into partials.
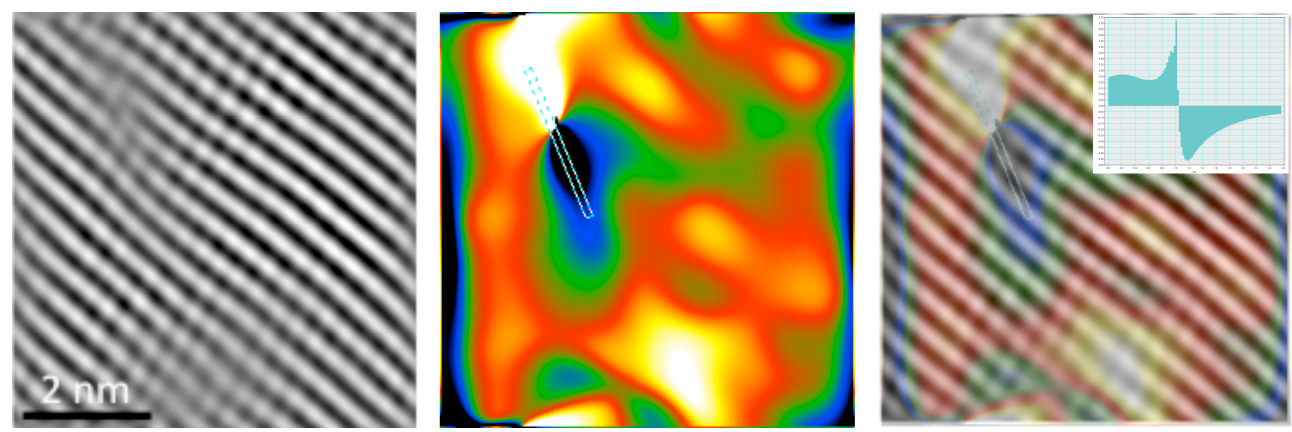

Fig.3 - Strain mapping around edge dislocation, showing switching in stress state (compression to tension) upon crossing dislocation core (inset). 